Health Sciences Mall, Vancouver, British Columbia V6T 1Z3, Canada. Phone: (604) 822-0908; Fax: (604) 822-5348; E-mail: cbrown@interchange.ubc.ca.

1. Lyon, M.F. 1961. Gene action in the X-chromosome of the mouse (Mus musculus L.). Nature. 190:372-373.

2. Migeon, B.R. 2007. Females are mosaics: Xinactivation and sex differences in disease. Oxford University Press. New York, New York, USA. 296 pp.

3. Cattanach, B.M., and Williams, C.E. 1972. Evidence of non-random $\mathrm{X}$ chromosome activity in the mouse. Genet. Res. 19:229-240.

4. Avner, P., et al. 1998. Molecular correlates of the murine Xce locus. Genet. Res. 72:217-224.

5. Heard, E., and Disteche, C.M. 2006. Dosage compensation in mammals: fine-tuning the expression of the X chromosome. Genes Dev 20:1848-1867.

6. Bolduc, V., et al. 2008. No evidence that skewing of $\mathrm{X}$ chromosome inactivation patterns is transmitted to offspring in humans. J. Clin. Invest. 118:333-341.

7. Linder, D., and Gartler, S.M. 1965. Glucose-6phosphate dehydrogenase mosaicism: Utilization as a cell marker in the study of leiomyomas. Science. 150:67-69.

8. Puck, J.M., Nussbaum, R.L., and Conley, M.E. 1987. Carrier detection in X-linked severe combined immunodeficiency based on patterns of X chromosome inactivation. J. Clin. Invest. 79:1395-1400.

9. Dobrovolny, R., et al. 2005. Relationship between
$\mathrm{X}$-inactivation and clinical involvement in Fabry heterozygotes. Eleven novel mutations in the alpha-galactosidase A gene in the Czech and Slovak population. J. Mol. Med. 83:647-654.

10. Bretherick, K., Gair, J., and Robinson, W.P. 2005. The association of skewed X chromosome inactivation with aneuploidy in humans. Cytogenet. Genome Res. 111:260-265.

11. Ozbalkan, Z., et al. 2005. Skewed X chromosome inactivation in blood cells of women with scleroderma. Arthritis Rheum. 52:1564-1570.

12. Kristiansen, M., et al. 2002. High frequency of skewed $\mathrm{X}$ inactivation in young breast cancer patients. J. Med. Genet. 39:30-33.

13. Migeon, B.R. 2006. The role of X inactivation and cellular mosaicism in women's health and sex-specific diseases. JAMA. 295:1428-1433.

14. Amos-Landgraf, J.M., et al. 2006. X chromosomeinactivation patterns of 1,005 phenotypically unaffected females. Am. J. Hum. Genet. 79:493-499.

15. Hatakeyama, C., et al. 2004. The dynamics of Xinactivation skewing as women age. Clin. Genet. 66:327-332.

16. Sharp, A., Robinson, D., and Jacobs, P. 2000. Ageand tissue-specific variation of $\mathrm{X}$ chromosome inactivation ratios in normal women. Hum. Genet. 107:343-349.

17. Knudsen, G.P., et al. 2006. Increased skewing of X chromosome inactivation in Rett syndrome patients and their mothers. Eur. J. Hum. Genet. 14:1189-1194.

18. Cotter, P.D., et al. 1995. Late-onset X-linked sid- eroblastic anemia. Missense mutations in the erythroid delta-aminolevulinate synthase (ALAS2) gene in two pyridoxine-responsive patients initially diagnosed with acquired refractory anemia and ringed sideroblasts. J. Clin. Invest. 96:2090-2096.

19. Gale, R.E., Fielding, A.K., Harrison, C.N., and Linch, D.C. 1997. Acquired skewing of X-chromosome inactivation patterns in myeloid cells of the elderly suggests stochastic clonal loss with age. Br. J. Haematol. 98:512-519.

20. Kristiansen, M., et al. 2005. Twin study of genetic and aging effects on $\mathrm{X}$ chromosome inactivation. Eur. J. Hum. Genet. 13:599-606.

21. Vickers, M.A., McLeod, E., Spector, T.D., and Wilson, I.J. 2001. Assessment of mechanism of acquired skewed $\mathrm{X}$ inactivation by analysis of twins. Blood. 97:1274-1281.

22. International HapMap Project. http://www.hapmap.org.

23. Ropers, H.H., Wienker, T.F., Grimm, T., Schroetter, K., and Bender, K. 1977. Evidence for preferential $\mathrm{X}$-chromosome inactivation in a family with Fabry disease. Am. J. Hum. Genet. 29:361-370.

24. Bicocchi, M.P., et al. 2005. Familial nonrandom inactivation linked to the $\mathrm{X}$ inactivation centre in heterozygotes manifesting haemophilia A. Eur. J. Hum. Genet. 13:635-640.

25. Plenge, R.M., et al. 1997. A promoter mutation in the XIST gene in two unrelated families with skewed X-chromosome inactivation. Nat. Genet. 17:353-356.

\title{
Blood is thicker than lymph
}

\author{
Mark L. Kahn \\ Department of Medicine and University of Pennsylvania Cardiovascular Institute, University of Pennsylvania, Philadelphia, Pennsylvania, USA.
}

\begin{abstract}
Growth of blood and lymphatic vessels is essential in the developing embryo and in the pathogenesis of human diseases such as cancer, but the signaling pathways that regulate vessel growth and function are not yet well characterized. In this issue of the JCI, studies by Fritz-Six et al. and Ichikawa-Shindo et al. demonstrate that loss of signaling by the adrenomedullin peptide results in embryonic edema and death (see the related articles beginning on pages 40 and 29, respectively). Remarkably, this phenotype is attributed to defects in lymphatic vessels by one group and to defects in blood vessels by the other. In addition to defining what I believe to be a novel angiogenic signaling pathway, these studies demonstrate the intricate molecular, genetic, and physiologic relationships between blood and lymphatic vessels that must be considered by investigators of vascular biology.
\end{abstract}

\section{Blood and lymphatic vessels: embryonic connections}

Mammals have 2 distinct vascular systems: a blood vascular network essential for the delivery of oxygen and nutrients, and a lymphatic vascular network that returns extracellular

Nonstandard abbreviations used: AM, adrenomedullin; CALCRL, calcitonin receptor-like receptor; CGRP, calcitonin gene-related peptide; RAMP, receptor activity-modifying protein.

Conflict of interest: The author has declared that no conflict of interest exists.

Citation for this article: J. Clin. Invest. 118:23-26 (2008). doi:10.1172/JCI34485. fluid and proteins to the blood circulation and coordinates the immune response. These vascular systems form sequentially during embryonic life. The blood vascular system develops first, coordinately with development of the heart and blood to feed the growing embryo. The lymphatic vascular system develops in midgestation as the blood cardiovascular system matures and hemodynamic forces rise (1). Mouse genetic studies have confirmed Florence Sabin's century-old hypothesis that lymphatic vessels arise from venous endothelial cells that exit the cardinal vein and coalesce to form the lymph sacs, the first lymphatic vascular structures $(2,3)$. Angiogenic sprouting from the primitive lymph sacs ultimately gives rise to a new vascular network of lymphatic vessels. Blood and lymphatic vessels are often considered as distinct systems with little overlapping function or regulation, but studies of genetically modified mice, and even the origin of human diseases such as Kaposi sarcoma (4, 5), have begun to reveal the strong molecular and physiologic relationships between these vascular entities. In this issue of the JCI, 2 studies of adrenomedullin (AM) signaling identify a developmental link between the blood and lymphatic vascular systems $(6,7)$.

\section{AM: a pleiotropic regulator of vascular function}

$\mathrm{AM}$ is a 52-aa peptide, first isolated from a human pheochromocytoma, that is a member of the family of calcitonin gene-related peptides (CGRPs), which is composed of calcitonin, AM, CGRP1 and CHRP2, and amylin. At the time of its discovery AM was noted to be a potent vasodilator (8), and it has subsequently been linked to the regulation of numerous vascular responses, 

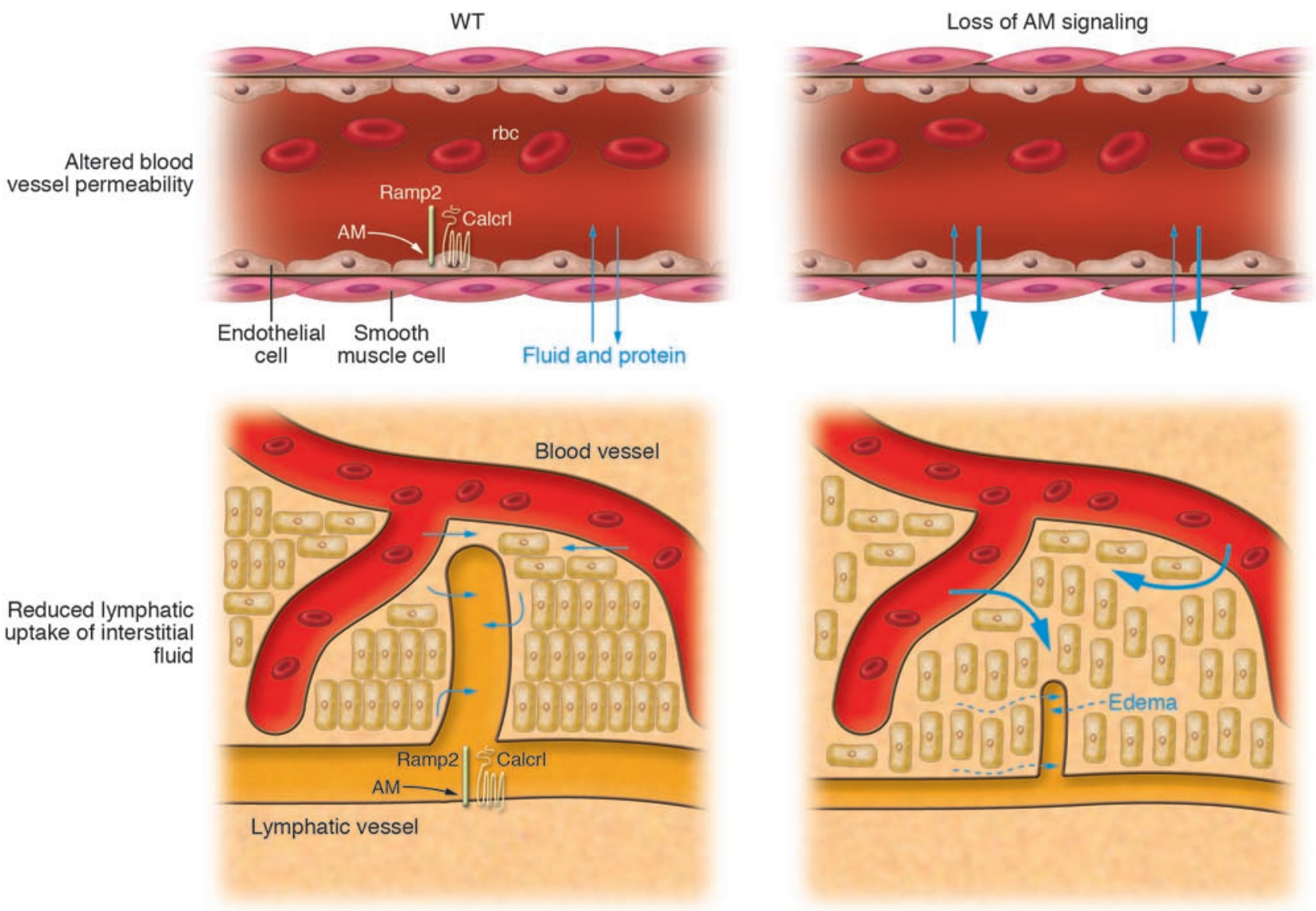

\section{Figure 1}

Development of edema due to loss of AM signaling. As reported by Fritz-Six et al. (6) and Ichikawa-Shindo et al. (7) in this issue of the $\mathrm{JCl}$, the massive edema observed in midgestation mouse embryos lacking AM signaling is caused by a combination of fluid and protein leakage from blood vessels due to altered vascular permeability (top panels) and reduced lymphatic uptake of extravasated fluid and protein due to reduced lymphatic vessel growth (bottom panels). In both blood and lymphatic vessels, AM signaling is mediated by Calcrl-Ramp2 receptor complexes expressed on endothelial cells. Arrows indicate the movement of fluid in response to hydrostatic pressure (an outward force in blood capillaries) and osmotic pressure (an inward force in blood capillaries).

including blood pressure, salt and water intake, and angiogenesis (reviewed in ref. 9). AM circulates in the blood, and its levels are elevated in many settings of cardiovascular stress, including hypertension, renal failure, congestive heart failure, pregnancy, and septic shock. These findings have raised interest in AM as a potential therapeutic target, but its biological role remains poorly defined.

The biology of AM is also complex at the molecular level. AM and other calcitonin family peptides are amidated at their $\mathrm{C}$ terminus and form a ring structure through disulfide bonds, features essential for their biological activity (10). The peptides are ligands for $\mathrm{G}$ protein-coupled receptors (GPCRs), but ligand-receptor interactions, and perhaps other aspects of receptor signaling, are strictly regulated by receptor activity-modifying proteins (RAMPs), single pass transmembrane proteins that associate with
GPCRs (11). RAMPs modulate the ligand binding of the calcitonin receptor-like receptor (CALCRL) such that RAMP1-CALCRL functions primarily as a CGRP receptor and RAMP2/3-CALCRL functions primarily as an AM receptor (11). The net effect of this tortuous path from ligand to signal is the potential for tremendous combinatorial diversity: the biological role of AM may be influenced by expression of the AM peptide; by expression of "competing" CGRPs; by expression of specific RAMP proteins; and by expression of CALCRL. Thus the role of AM in any given tissue or physiologic response is not easily predicted.

\section{Dissecting AM function: genetic studies}

To understand the biological role of AM, 2 groups have been pursuing genetic studies in mice, the most recent of which are described in this issue of the JCI $(6,7)$. Previous research by these groups has shown that AM-deficient mice die in midgestation with vascular defects, but the precise nature of these defects has been unclear. Caron et al. reported severe edema as well as thinner arterial walls and cardiac defects in AMdeficient embryos (12), while Shindo et al. reported diffuse hemorrhage associated with structural defects in blood vessels and only occasional edema (13). Whether these lethal phenotypes arise due to heart failure, vascular leak and hemorrhage, or primary lymphatic vessel defects has been uncertain. Here these investigators present new findings addressing the role of AM and its receptors RAMP2 and CALCRL $(6,7)$. Remarkably, although these 2 groups now report highly concordant phenotypes, they reach different conclusions regarding the mechanism by which they arise. 
Fritz-Six et al. (6) report that deletion of $\mathrm{AM}$, Calcrl, or Ramp2 results in massive cutaneous edema and midgestation lethality in mice. This group describes a defect in lymphatic vascular development in these animals characterized by retarded growth of the deeper lymphatics in the neck region (the jugular lymph sacs), a site where the first lymphatic vessels arise from venous endothelial precursors. Ultrastructural analysis of lymphatic vessels in Ramp2 $2^{-/-}$ embryos demonstrates intact tight junctions but thin and occasionally dead endothelial cells. Significantly, the authors demonstrate that AM-Ramp2/Calcrl signaling drives lymphatic endothelial proliferation in vitro, identifying AM as what they believe to be a novel lymphangiogenic growth factor. These findings in cultured endothelial cells are supported by the development of a similar phenotype in mice in which the Calcrl receptor has been deleted in endothelial cells (and to some extent in hematopoietic cells) by a Tie2Cre transgene. These authors therefore attribute the edema and death of embryos lacking AM, Ramp2, or Calcrl to defects in lymphatic vessel growth.

Ichikawa-Shindo et al. (7) use nearly identical genetic experiments to reach different conclusions regarding the role of AM signaling. They describe generalized edema and midgestation death, as well as occasional hemorrhage, in Ramp2-deficient animals. They detect Ramp 2 expression in the endothelial cells of developing blood vessels and histologic defects in Ramp2 $2^{-/-}$blood vessels, including reduced arterial smooth muscle cell coverage and thinned, detached endothelial cells. Gene expression analysis reveals reduced levels of endothelial adhesive genes in the absence of Ramp2, while in vitro studies suggest that increased AM signaling reduces endothelial permeability by tightening intercellular junctions. These findings are supported by elegant studies of Ramp $2^{+/-}$mice demonstrating changes in blood pressure, blood vessel angiogenesis, and vascular permeability. The authors conclude that AM serves critical roles in blood vessels, including regulation of vascular stability and permeability, and that embryos lacking AM signaling die due to leaky and unstable blood vessels.

\section{Blood and lymphatic roles for AM: a} genetic test of the Starling principle?

That 2 groups could study the same mice and reach such different conclusions regarding the biological role of AM sig- naling $(6,7)$ provides instructive insight into the study of vascular biology. Importantly, there are fundamental points of agreement between the studies: embryos lacking AM, Ramp2, or Calcrl develop severe edema and die in midgestation; deficient vessels exhibit ultrastructural vascular defects; and AM signaling regulates endothelial responses ex vivo. These findings, and the studies by Fritz-Six et al. of conditionally deleted Calcrl embryos (6), support the conclusion that AM signaling through Ramp2/Calcrl receptors is required in endothelial cells of the developing embryo (Figure 1). What, then, is the critical role of this pathway in the developing vasculature - lymphatic vessel growth or blood vessel stabilization? Not surprisingly, these roles are not mutually exclusive and even overlap physiologically. More surprising is that the questions posed by these studies harken back to physiologic studies performed over a century ago by E.H. Starling to understand the origin of lymph (14).

The 2 most striking phenotypes in embryos lacking AM signaling are massive edema and midgestation death. Edema results from the accumulation of extracellular fluid due to either excess leakage from the blood or failure of lymphatic vessels to return fluid from the interstitium. Starling proposed in 1896 that fluid exchange between the blood and the tissues is balanced by differences in hydrostatic pressure (favoring movement from the blood to the tissues) and osmotic pressure (favoring movement from the tissues to the blood) and predicted that the amount of fluid that must be returned by lymphatics to prevent edema is normally minimal because a near equilibrium exists between these forces (14). While specifics of the Starling principle have since been modified (15), the net effect is a very low rate of fluid filtration from the blood and a correspondingly low level of lymph flow (only $120 \mathrm{ml} / \mathrm{h}$ in an adult). Starling's principle suggests that loss of lymphatic function alone would not result in the rapid development of massive edema and death, and more recent genetic studies in mice support this conclusion. Loss of VEGF-C, an essential lymphatic endothelial growth factor, results in a more severe lymphatic defect than that seen in mice lacking AM signaling but permits longer embryonic survival with the same or less edema (16). Thus loss of AM signaling must affect the blood vasculature to increase the move- ment of fluid from the blood to the tissues in addition to disrupting lymphatic development. Human cases of fetal hydrops (a condition defined by generalized soft tissue edema) most frequently arise as a result of congestive heart failure and increased capillary hydrostatic pressure (17). While this mechanism has not been excluded in embryos lacking AM signaling (in fact, cardiac defects are a notable feature of embryos lacking this pathway; refs. 12,18$)$, the endothelial permeability and vascular integrity defects described by Ichikawa-Shindo et al. (7) provide a direct explanation for increased fluid movement from the blood as well as for the hemorrhage observed. Combined with the lymphatic defects demonstrated by Fritz-Six et al. (6), these findings suggest that loss of AM signaling is an ideal recipe for edema (Figure 1).

\section{AM signaling: lessons and future directions}

The studies of AM signaling reported in this issue of the JCI $(6,7)$ identify a signaling pathway required for regulation of both blood and lymphatic vessel development and highlight many of the challenges faced by investigators of vascular biology. The blood and lymphatic vascular systems may only be physically connected where the thoracic duct empties into the subclavian vein, but they are genetically, molecularly, and physiologically connected at many other levels. Loss of shared endothelial signaling pathways involving AM, angiopoietin (19), and ephrin B2 (20) produce phenotypes in both systems. Dissecting the roles of signaling pathways in blood and lymphatic vessels therefore requires molecular and genetic tools specific to each system. Molecular markers of the lymphatic vasculature have been available for only a decade $(21,22)$, and lymphatic-specific genetic tools are still being developed. In addition, the vasculature of both networks is highly heterogeneous, and disorders of some regions, such as the microcirculation, may result in dramatic physiologic perturbations that are not accompanied by easily detectable histologic abnormalities. The well-developed physiologic tools used daily for clinical evaluation of cardiovascular disorders, such as intravascular hemodynamic measurements, are not yet applicable to developing model organisms such as the mouse or fish. Finally, although it is tempting to predict that vascular roles defined in the developing embryo are 
conserved in mature animals and human disease states, clear demonstration of the validity of such predictions is often lacking and requires complex genetic and physiologic approaches. The studies in this issue of the JCI identify AM signaling as a key regulator of blood and lymphatic vessel function, but whether Starling would be disappointed or gratified to learn of the persistent significance of his experiments in our molecular and genetic age is not clear. Clear definition of the vascular roles of AM signaling will parallel progress in addressing these broad issues in vascular biology, leaving plenty of future work for these and other investigators.

\section{Acknowledgments}

I thank the members of my laboratory and Guillermo Oliver for interesting discussions.

Address correspondence to: Mark L. Kahn, Department of Medicine, University of Pennsylvania, 952 BRB II/III, 421 Curie Blvd., Philadelphia, Pennsylvania 19104, USA. Phone: (215) 898-9007; Fax: (215) 573-2094; E-mail: markkahn@mail. med.upenn.edu.
1. Adams, R.H., and Alitalo, K. 2007. Molecular regulation of angiogenesis and lymphangiogenesis. Nat. Rev. Mol. Cell Biol. 8:464-478.

2. Sabin, F. 1901. On the origin of the lymphatic system from the veins and the development of the lymph hearts and thoracic duct in the pig. Am.J. Anat. 4:367-389.

3. Srinivasan, R.S., et al. 2007. Lineage tracing demonstrates the venous origin of the mammalian lymphatic vasculature. Genes Dev. 21:2422-2432.

4. Wang, H.W., et al. 2004. Kaposi sarcoma herpesvirus-induced cellular reprogramming contributes to the lymphatic endothelial gene expression in Kaposi sarcoma. Nat. Genet. 36:687-693.

5. Hong, Y.K., et al. 2004. Lymphatic reprogramming of blood vascular endothelium by Kaposi sarcomaassociated herpesvirus. Nat. Genet. 36:683-685.

6. Fritz-Six, K.L., Dunworth, W.P., Li, M., and Caron, K.M. 2008. Adrenomedullin signaling is necessary for murine lymphatic vascular development. J. Clin. Invest. 118:40-50.

7. Ichikawa-Shindo, Y., et al. 2008. The GPCR modulator protein RAMP2 is essential for angiogenesis and vascular integrity. J. Clin. Invest. 118:29-39.

8. Kitamura, K., et al. 1993. Adrenomedullin: a novel hypotensive peptide isolated from human pheochromocytoma. Biochem. Biophys. Res. Commun. 192:553-560.

9. Gibbons, C., Dackor, R., Dunworth, W., Fritz-Six, K., and Caron, K.M. 2007. Receptor activity-modifying proteins: RAMPing up adrenomedullin signaling. Mol. Endocrinol. 21:783-796.

10. Czyzyk, T.A., et al. 2005. Deletion of peptide amidation enzymatic activity leads to edema and embryonic lethality in the mouse. Dev. Biol. 287:301-313.

11. McLatchie, L.M., et al. 1998. RAMPs regulate the transport and ligand specificity of the calcitoninreceptor-like receptor. Nature. 393:333-339.
12. Caron, K.M., and Smithies, O. 2001. Extreme hydrops fetalis and cardiovascular abnormalities in mice lacking a functional Adrenomedullin gene. Proc. Natl. Acad. Sci. U. S. A. 98:615-619.

13. Shindo, T., et al. 2001. Vascular abnormalities and elevated blood pressure in mice lacking adrenomedullin gene. Circulation. 104:1964-1971.

14. Starling, E.H. 1896. On the absorption of fluids from connective tissue spaces. J. Physiol. Lond. 19:312-326.

15. Michel, C.C. 1997. Starling: the formulation of his hypothesis of microvascular fluid exchange and its significance after 100 years. Exp. Physiol. 82:1-30.

16. Karkkainen, M.J., et al. 2004. Vascular endothelial growth factor $\mathrm{C}$ is required for sprouting of the first lymphatic vessels from embryonic veins. Nat. Immunol. 5:74-80.

17. Machin, G.A. 1989. Hydrops revisited: literature review of 1,414 cases published in the 1980 s. Am. J. Med. Genet. 34:366-390.

18. Dackor, R.T., et al. 2006. Hydrops fetalis, cardiovascular defects, and embryonic lethality in mice lacking the calcitonin receptor-like receptor gene. Mol. Cell. Biol. 26:2511-2518.

19. Gale, N., et al. 2002. Angiopoietin-2 is required for postnatal angiogenesis and lymphatic patterning, and only the latter role is rescued by angiopoietin-1. Dev. Cell. 3:411.

20. Makinen, T., et al. 2005. PDZ interaction site in ephrinB2 is required for the remodeling of lymphatic vasculature. Genes Dev. 19:397-410.

21. Wigle, J.T., and Oliver, G. 1999. Prox1 function is required for the development of the murine lymphatic system. Cell. 98:769-778.

22. Banerji, S., et al. 1999. LYVE-1, a new homologue of the CD44 glycoprotein, is a lymph-specific receptor for hyaluronan. J. Cell Biol. 144:789-801.

\title{
Anticonvulsant effects of leptin in epilepsy
}

\author{
Sabrina Diano1,2 and Tamas L. Horvath ${ }^{1,2,3}$ \\ 1Department of Obstetrics, Gynecology \& Reproductive Sciences, ${ }^{2}$ Department of Neurobiology, and \\ ${ }^{3}$ Section of Comparative Medicine, Yale School of Medicine, New Haven, Connecticut, USA.
}

\begin{abstract}
Secreted from adipose tissue at levels proportional to fat stores, the hormone leptin is a critical regulator of the hypothalamic machinery that controls feeding and energy metabolism. Despite the critical role of leptin in the maintenance of energy homeostasis, no leptin-based therapeutic approaches have emerged to combat metabolic disorders such as obesity or diabetes. In this issue of the JCI, Xu et al. report a robust influence of leptin, beyond its role in metabolism, on hippocampal neuronal processes implicated in the etiology of epileptic seizures, learning, and memory (see the related article beginning on page 272). They show, in two rodent seizure models, that leptin administered directly to the brain or nasal epithelium suppresses seizures via direct effects on glutamate neurotransmission in the hippocampus. These observations suggest that leptin may have therapeutic potential in the treatment of epilepsy and strengthen the notion that peripheral metabolic hormones such as leptin play important roles in the regulation of higher brain functions.
\end{abstract}

Nonstandard abbreviations used: AMPA, $\alpha$-amino-3hydroxy-5-methyl-4-isoxazole proprionic acid.

Conflict of interest: The authors have declared that no conflict of interest exists.

Citation for this article: $J$. Clin. Invest. 118:26-28 (2008). doi:10.1172/JCI34511.
Over the last century, it has been hypothesized and then experimentally proven that a humoral signal from the periphery, specifically from adipose tissue, plays a role in the regulation of feeding and energy metabolism (1-5). The identity of this hormone, named leptin, was elegantly revealed by Friedman and colleagues in 1994 (6), and thus began a new era in the use of molecular biology in the quest to identify a cure for metabolic disorders, most notably obesity. This discovery revolutionized the field and attracted increasing numbers of creative researchers with sophisticated tools in an effort to determine the mechanisms of action of leptin in the hypothalamus and its role in the regulation of feeding and adiposity (reviewed in ref. 7). To date, despite the great wealth of knowledge that has been gained and the discovery of new hormones, no leptin-based medical remedy has emerged for the treatment of obesity. While new avenues of research in this area are being pursued by many, the study by $\mathrm{Xu}$ et al. reported in this issue of the JCI (8) reveals that the leptin signaling pathway has important functions that go beyond 